PROCEEDINGS OF THE

AMERICAN MATHEMATICAL SOCIETY

Volume 98, Number 4, December 1986

\title{
SOME REMARKS ON AFFINE RINGS
}

\author{
S. MONTGOMERY AND L. W. SMALL ${ }^{1}$
}

\begin{abstract}
Various topics on affine rings are considered, such as the relationship between Gelfand-Kirillov dimension and Krull dimension, and when a "locally affine" algebra is affine. The dimension result is applied to study prime ideals in fixed rings of finite groups, and in identity components of group-graded rings.
\end{abstract}

0. Introduction. We study affine rings (i.e., rings finitely generated as algebras over a central subring) and group actions on them. Of particular interest is the relationship between the Gelfand-Kirillov (GK) dimension and the classical Krull dimension. Our first theorem shows for affine prime PI rings over a field that the GK dimension of a prime subalgebra is the same as its Krull dimension. This result is then applied to give a rapid proof of a result of Alev [1], on prime ideals in fixed rings. Later we turn to group-graded affine rings deriving results similar to those previously obtained for group actions; the notion of equivalence is introduced for prime ideals in the identity component, and an analog of Alev's theorem is proved. We also consider when a prime PI algebra which is "locally affine" must be affine.

Some of the results in this paper (including Theorem 1) were announced at the NATO A.S.I. in Ring Theory, Antwerp, 1983 (see [7]).

1. Prime subrings of PI rings. We begin by stating a form of the Artin-Tate lemma, which will be used throughout this paper.

Lemma 1. Let $R \subset S$ be algebras over a commutative Noetherian ring $C$, such that $S$ is a finite module over $R$ and affine over $C$. If $R$ is contained in the center of $S$, then $R$ is $C$-affine. Consequently $R$ is Noetherian and $S$ is a Noetherian $R$-module.

Proof. The usual commutative argument works in this situation; see also Lemma 27 of [11].

Our first theorem extends a result of Malliavin [4] on affine PI algebras to prime subrings of such rings, which are not necessarily affine. For any algebra $A$ over a field $k$, we let $\operatorname{cl}(A)$ denote the classical Krull dimension of $A$, and $\operatorname{GK}(A)$ denote the Gelfand-Kirillov dimension of $A$.

TheOREM 1. Let $A$ be a prime PI algebra which is affine over a field $k$. If $B$ is a prime subalgebra of $A$, then $\operatorname{GK}(B)=\operatorname{cl}(B)$.

Received by the editors March 25, 1985 and, in revised form, August 19, 1985.

1980 Mathematics Subject Classification (1985 Revision). Primary 16A38, 16 A72.

${ }^{1}$ Both authors acknowledge support from the NSF. 
Proof. When $A$ is commutative, the theorem seems to be known. However, since we were unable to find a proof in the literature, we provide one for completeness. In this case, we must show that $\operatorname{cl}(B)=\operatorname{tr} \operatorname{deg}_{k}(B)$, since transcendence degree coincides with GK dimension if $B$ is commutative.

We proceed by induction on $\operatorname{tr} \operatorname{deg}_{k} A$. If $A$ is algebraic over $k$, then $A$ (and so $B$ ) is finite dimensional over $k$. Since $B$ is a domain, it is a field. Thus $\operatorname{cl}(B)=0=$ $\operatorname{tr} \operatorname{deg}_{k}(B)$. Now if there exists a prime ideal $P \neq 0$ in $A$ with $P \cap B=(0)$, then $B$ is contained in $A / P$, which has lower transcendence degree than $A$ (since it is well known that for an affine commutative domain, $\operatorname{cl}(A)=\operatorname{tr} \operatorname{deg} A)$. We would then be done by induction. Thus we may assume that $P \cap B \neq 0$, for any prime ideal $P$ of A.

It follows that $Q(A)$ is algebraic over $Q(B)$, where $Q(A)$ and $Q(B)$ are the respective quotient fields of $A$ and $B$. For, the assumption about primes implies that $Q(A)=A S^{-1}$, where $S=B-\{0\}$. Then $A S^{-1}$ is affine over $Q(B)$, and is a field; thus by the Nullstellensatz, $A S^{-1}=Q(A)$ is algebraic over $Q(B)$.

If $A=k\left[\alpha_{1}, \ldots, \alpha_{r}\right]$, let $s$ be a common denominator in $B$ for the coefficients of the monic minimal polynomials of the $\alpha_{i}$ over $Q(B)$. Then $A[1 / s]$ is integral over $B[1 / s]$ (note that by the Artin-Tate lemma, this says $B[1 / s]$ is affine). Thus

$$
\operatorname{cl}(B) \geqslant \operatorname{cl}(B[1 / s])=\operatorname{cl}(A[1 / s])=\operatorname{tr} \operatorname{deg}_{k}(A) \geqslant \operatorname{trdeg}(B) .
$$

Since it is always true that $\operatorname{cl}(B) \leqslant \operatorname{tr} \operatorname{deg}(B)$, the commutative case is proved.

Now consider $Z(B)$, the center of $B$. We claim that $Z(B)$ is contained in a commutative affine $k$-algebra, namely the algebra $Z(B) Z(T(A))$, where $T(A)$ is the trace ring of $A$. For, it is known that $T(A)$ is affine and a finite $Z(T(A))$-module [8, Proposition 3]. Thus by Lemma $1, Z(T(A))$ is Noetherian and $T(A)$ is a Noetherian $Z(T(A))$-module. Since $Z(B) Z(T(A)) \subseteq T(A)$, it is affine, proving the claim. Thus by the commutative case, $\operatorname{cl}(Z(B))=\mathrm{GK}(Z(B))$.

Now choose $c$ in the Formanek center of $B$, so that $B[1 / c]$, the localization of $B$ at the set $\left\{c^{n}\right.$, all $\left.n \geqslant 1\right\}$, is an Azumaya algebra over $Z(B)[1 / c]$ (by Artin's theorem). Since $B[1 / c]$ is also a prime subring of an affine algebra, the above argument applies to $Z(B)[1 / c]$. Moreover, GK dimension is preserved under central localization. Thus

$$
\operatorname{cl}(B) \geqslant \operatorname{cl}(B[1 / c])=\operatorname{cl}(Z(B)[1 / c])=\operatorname{GK}(Z(B)[1 / c])=\operatorname{GK}(Z(B)) .
$$

Since $B$ is a prime PI ring, $\operatorname{GK}(B)=\operatorname{GK}(Z(B))$ by [11, Theorem 45]. Thus $\operatorname{cl}(B) \geqslant \operatorname{GK}(B)$.

On the other hand, $\operatorname{cl}(B) \leqslant \mathrm{GK}(B)$; for, if $R$ is any $k$-algebra and $I$ an ideal of $R$ containing a regular element, then $\mathrm{GK}(R / I) \leqslant \mathrm{GK}(R)-1$. Thus $\operatorname{cl}(B)=\mathrm{GK}(B)$.

Both R. Guralnick and D. Saltman have pointed out to us that the proof of the theorem characterizes prime subrings of affine PI algebras.

COROllaRY 1. Let $B$ be a prime PI algebra over $k$. Then $B$ can be imbedded in some affine prime $P I$ algebra $A$ over $k$ if and only if there exists $z \neq 0$ in the center of $B$ such that the localization $B[1 / z]$ is affine. 
Proof. It suffices to prove the "only if" direction. Thus, let $B$ be a prime subalgebra of an affine prime PI algebra $A$. If $A$ is commutative, the proof of Theorem 1 gives that $B[1 / s]$ is affine. Thus assume $A$ is not commutative.

In this case the proof shows that $B[1 / c]$ is an Azumaya algebra (and thus a finite module) over $Z(B)[1 / c]$. Moreover, $Z(B)$ is contained in a commutative affine $k$-algebra, so by the commutative case $Z(B)[1 / s]$ is affine for some $s$. Then $B[1 / c s]=B[1 / c][1 / s]$ is a finite module over $Z(B)[1 / s][1 / c]$, an affine algebra. Thus $B[1 / c s]$ is affine.

2. Prime ideals in fixed rings. In this section we will apply Theorem 1 to give a new proof of a result of Alev [1], which we believe to be more straightforward.

For any ring $S$ with idempotent $e \neq 0$, the mapping $\Phi: S \rightarrow e S e$ induces a map from ideals of $S$ to ideals of $e S e$. Moreover if $\operatorname{Spec}_{e} S$ denotes the primes of $S$ not containing $e$, then the induced map

$$
\Phi: \operatorname{Spec}_{e} S \rightarrow \text { Spec } e S e
$$

is a bijection.

In the situation of a finite group $G$ of automorphisms of a ring $R$, with $|G|^{-1} \in R$, it is known that the fixed ring $R^{G} \cong e(R * G) e$, where $R * G$ denotes the skew group ring and $e=|G|^{-1} \Sigma_{g} g \in R * G$. Thus there is a bijection $\Phi: \operatorname{Spec}_{e}(R * G) \rightarrow \operatorname{Spec}^{G}$ where we have identified $R^{G}$ with $e(R * G) e$.

We can now give our proof of Alev's theorem [1].

THEOREM 2. Let $R$ be an affine PI algebra, $G$ a finite group of automorphisms of $R$ with $|G|^{-1} \in R$, and $p \in \operatorname{Spec}\left(R^{G}\right)$. Then

$$
\operatorname{GK}\left(R^{G} / p\right)=\operatorname{cl}\left(R^{G} / p\right) .
$$

Proof. Since $R$ is an affine PI algebra, the skew group ring $R * G$ is also an affine PI algebra. For $p \in \operatorname{Spec} R^{G}$, let $\mathscr{P}=\Phi^{-1}(p) \in \operatorname{Spec} R * G$. Then $A=R * G / \mathscr{P}$ is a prime affine PI algebra.

Denoting the image of $e$ in $A$ by $\bar{e}$, it follows that $\bar{e} A \bar{e} \cong e(R * G) e / e \mathscr{P} e \cong$ $R^{G} / p=B$. Now apply Theorem 1 .

Continuing with our assumption that $|G|^{-1} \in R$, recall from [5] that two primes $p, q$ of $R^{G}$ are equivalent if $\Phi^{-1}(p) \cap R=\Phi^{-1}(q) \cap R$. It is shown there that $p \sim q$ if and only if there exists a prime $P$ of $R$ such that $p$ and $q$ are minimal over $P \cap R^{G}$; moreover $P$ is unique up to its $G$-orbit in $R$.

COROllaRY 2. Let $R$ be an affine PI algebra, and $G$ a finite group of automorphisms of $R$ with $|G|^{-1} \in R$. If $p \sim q$ in $\operatorname{Spec} R^{G}$, then $\operatorname{cl}\left(R^{G} / p\right)=\operatorname{cl}\left(R^{G} / q\right)$.

Proof. The proof is immediate from Theorem 2 and a result of the present authors [9] that $\mathrm{GK}\left(R^{G} / p\right)=\mathrm{GK}\left(R^{G} / q\right)$.

The conclusion of the Corollary may be restated as saying that depth is an "equivalence invariant" for primes of $R^{G}$. This is false if $R$ is not affine, as the following example (announced in [7]) shows:

EXAMPLE 1. Let $D$ be a commutative valuation domain of rank 2 which is an algebra over a field $k$ of characteristic $\neq 2$. Let the prime ideals of $D$ be $M \supset P \supset$ 
(0), where $M$ is maximal, and let $R=\left({ }_{P}^{k+P} \underset{D}{D}\right)$. Let $G=\langle g\rangle$, where $g$ is conjugation by $\left(\begin{array}{cc}1 & 0 \\ 0 & -1\end{array}\right)$. Then $R^{G} \cong(k+P) \oplus D$. Let $p=(0, D)$ and $q=(k+P, 0)$; then $p \sim q$ in $R^{G}$, since they are both minimal over (0). However, $\operatorname{cl}\left(R^{G} / p\right)=\operatorname{cl}(k+P)$ $=1$ and $\operatorname{cl}\left(R^{G} / p\right)=\operatorname{cl}(D)=2$.

3. Locally affine PI algebras. If $R$ is a commutative domain, not a field, which is an algebra over a field $k$, then the following is known: If the localization of $R$ at every nonzero non-unit of $R$ is $k$-affine, then $R$ itself is $k$-affine. We wish to thank D. Farkas and R. Guralnick, who provided us with proofs of this fact.

The natural question then arises as to whether this result extends to prime PI algebras by considering only localizations at the nonzero non-units in the Formanek center of $R$. We shall see below that this is false.

We require another consequence of Lemma 1, which is a slight extension of Noether's theorem.

COROllaRY 3. Let $R$ be an affine algebra over a commutative Noetherian ring $C$, and say that $R$ is a finite module over its center $Z$. Let $G$ be a finite group of $C$-automorphisms of $R$. Then $R^{G}$ is affine over $C$.

Proof. By Lemma $1, Z$ is $C$-affine and Noetherian. Since $Z$ is $G$-stable, Noether's theorem applies to $Z$; thus $Z^{G}$ is affine and Noetherian, and $\left[Z: Z^{G}\right]<\infty$. Thus $R$ is finite over $Z^{G}$, so is a Noetherian $Z^{G}$-module. Since $Z^{G} \subset R^{G} \subset R$, it follows that $R^{G}$ is affine over $C$.

EXAmple 2. Let $U=k\{X, Y\}$ denote the ring generated by two generic $2 \times 2$ matrices over a field $k$ of characteristic $\neq 2$. Define $g \in \operatorname{Aut}(U)$ by $X^{g}=X$, $Y^{g}=-Y$. Then if $G=\langle g\rangle$, it is known that $U^{G}$ is not finitely generated (this is due to Formanek; see [6]). Also, $U^{G}$ has the same PI degree as $U$, since it is easy to see that $U^{G}$ is not commutative (since $X, Y^{2} \in U^{G}$, if $X Y^{2}=Y^{2} X$ then $2 \times 2$ matrices would satisfy an identity of degree 3 , a contradiction). Thus for any $t$ in the Formanek center of $U^{G}, t$ is also in the Formanek center of $U$.

Now let $R=U^{G}$. The localization $A=U[1 / t]$ of $U$ at $t$ is an Azumaya algebra by Artin's theorem, and it is clearly $k$-affine. Thus $A^{G}$ is affine by Corollary 3 . But $A^{G}=U^{G}[1 / t]=R[1 / t]$. Thus, although $R$ is not affine, $R[1 / t]$ is affine for all such $t$.

However, there is a correct generalization of the commutative result. Guralnick's argument extends to prime PI rings if we consider all of the non-units in the center.

TheOREM 3 (R. GURALNICK). Let $R$ be a prime PI algebra with 1 over a field $k$, and assume that $Z(R)$ is not a field (that is, $R$ is not simple). If $R[1 / a]$ is affine over $k$ for all nonzero non-units $a$ in $Z(R)$, then $R$ is affine over $k$.

Proof. Write $Z=Z(R)$, and let $I=\{a \in Z \mid a=0$ or $R[1 / a]$ is affine $\}$. Clearly, it suffices to prove that $I=Z$, for then $R=R[1 / 1]$ is affine. To do this, we first show that $I$ is a nonzero ideal of $Z$ which contains all non-units of $Z$, and then show that $Z$ is not local (forcing $I=Z$ ). 
First, our hypotheses give that $I \neq 0$ and that all non-units are in $I$. Let $0 \neq a \in I$ and $0 \neq b \in Z$. then $R[1 / a b]=R[1 / a][1 / b]$ is affine, so $a b \in I$. Suppose $0 \neq a b$ with $a, b \in I$ and $a+b \neq 0$. Then since $R[1 / a]$ and $R[1 / b]$ are affine, we can choose $\alpha_{1}, \ldots, \alpha_{t} \in R$ so that if $S=k\left[\alpha_{1}, \ldots, \alpha_{t}\right]$, then $R[1 / a]=$ $S[1 / a]$ and $R[1 / b]=S[1 / b]$. Thus if $\beta \in R$, there exists some positive integer $N$ with $a^{N} \beta, b^{N} \beta \in S$. Hence $(a+b)^{2 N} \beta \in S$, and so $R[1 /(a+b)]=S[1 /(a+b)]$ is affine. Thus $a+b \in I$, and $I$ is an ideal.

Now let $0 \neq a$ be a non-unit of $Z$, and let $0 \neq c$ be an element in the Formanek center of $R$. Then $R^{\prime}=R[1 / a c]$ is affine; by Artin's theorem, it is also an Azumaya algebra, since $c$ is a unit in $R^{\prime}$. Thus $Z^{\prime}=Z\left(R^{\prime}\right)=Z[1 / a c]$ is affine. It follows that

$$
\operatorname{cl}(Z) \leqslant \operatorname{tr} \operatorname{deg}_{k}(Z)=\operatorname{trdeg}(Z[1 / a c])=\operatorname{cl}(Z[1 / a c]) \leqslant \operatorname{cl}(Z) .
$$

Thus $\operatorname{cl}(Z)=\operatorname{cl}(Z[1 / a c])$, and so $Z$ is not local.

4. Rings graded by finite groups. We prove here analogs for graded rings of some of our results on fixed rings and prime ideals, using work of Cohen and Montgomery [2].

Let $A$ be a $k$-algebra graded by the finite group $G$; that is, $A=\sum_{g} \oplus A_{g}$, where each $A_{g}$ is a $k$-subspace and $A_{g} A_{h} \subseteq A_{g h}$. The identity component $A_{1}$ is a subring and plays the role of the fixed ring. We write elements of $A$ as $a=\sum a_{g}$, where $a_{g} \in A_{g}$.

Since $A$ is graded, $A$ is a $k[G]^{*}$-module algebra, where $k[G]^{*}=\operatorname{Hom}_{k}(k[G], k)$, and so we can form the smash product $A \# k[G]^{*}$. Concretely, $A \# k[G]^{*}$ is described as follows: fix as a basis for $k[G]^{*}$ the dual basis $\left\{p_{g}\right\}$ to the set $G$; that is, $p_{g}(h)=\delta_{g, h}, g, h \in G$. Then $A \# k[G]^{*}=A \otimes_{k} k[G]^{*}$ as a $k$-vector space, with multiplication given by $\left(a \# p_{g}\right)\left(b \# p_{h}\right)=a b_{g h^{-1}} \# p_{h}$. It follows that $p_{1}\left(A \# k[G]^{*}\right) p_{1}=p_{1} A_{1} \cong A_{1}[2$, Corollary 1.5]. This is the analog of the fact for group actions that $e(R * G) e \cong R^{G}$.

We can now prove a version of Noether's theorem for graded rings. We believe it is new, even for commutative rings.

THEOREM 4. Let $A$ be an affine k-algebra which is graded by the finite group $G$, where $k$ is a commutative Noetherian ring.

(1) If $A$ is also Noetherian, then $A_{1}$ is affine and Noetherian.

(2) If $A$ is a finite module over its center $Z$, then $A_{1}$ is affine and Noetherian, and $A$ is Noetherian.

Proof. (1) Since $A \# k[G]^{*}$ is generated over $A$ by the $\left\{1 \# p_{g}\right\}, A \# k[G]^{*}$ is also affine and Noetherian. Using $S=A \# k[G]^{*}$ and $e=p_{1}$, Corollary 1 of [8] applies to give $e S e \cong A_{1}$ affine over $k$. Similarly, $e S e$ is Noetherian. Alternatively, $A_{1}$ is Noetherian since the map $\pi: A \rightarrow A_{1}$ given by $a \rightarrow a_{1}$ is a projection onto $A_{1}$, so the lattice of right (left) ideals of $A_{1}$ can be imbedded in that of $A$.

(2) By Lemma $1, Z$ is $k$-affine and Noetherian, and $A$ is Noetherian. We are now done by (1).

We remark that if $A$ is commutative, the standard Artin-Tate lemma argument works, since $A$ is integral over $A_{1}$ by a result of Bergman (see [10]). 
The theorem is false if $A$ is not Noetherian:

ExAmple 3. $A$ prime, $P I$, affine $k$-algebra $A$, graded by $\mathbf{Z}_{2}$, such that $A_{1}$ is not affine. To see this, we note that for any ring $S$, the ring $R=M_{2}(S)$ of $2 \times 2$ matrices over $S$ is $\mathbf{Z}_{2}$-graded, namely $R_{1}=\left(\begin{array}{ll}S & 0 \\ 0 & S\end{array}\right)$ and $R_{g}=\left(\begin{array}{ll}0 & S \\ S & 0\end{array}\right)$ (where we have written $\mathbf{Z}_{2}$ multiplicatively as $\{1, g\}$ ). Thus we may simply use Example 2 of [8], which was an affine prime PI subring of $2 \times 2$ matrices such that its subring of diagonal elements was not affine.

Next we prove the analog of Theorem 2 for graded rings. As in the discussion before Theorem 2, with $e=p_{1}$ and $S=A \# k[G]^{*}$, let $\operatorname{Spec}_{e} A \# k[G]^{*}$ denote the set of prime ideals of $S$ which do not contain $e$. Then there is a bijection

$$
\Psi: \operatorname{Spec}_{e}\left(A \# k[G]^{*}\right) \rightarrow \operatorname{Spec} A_{1} .
$$

TheOREM 5. Let $A$ be an affine PI algebra over a field $k$, such that $A$ is graded by the finite group $G$, and say that $p \in \operatorname{Spec} A_{1}$. Then $\mathrm{GK}\left(A_{1} / p\right)=\operatorname{cl}\left(A_{1} / p\right)$.

Proof. Follow exactly the proof of Theorem 2, using $A \# k[G]^{*}, \Psi$, and $p_{1}$ in place of $R * G, \Phi$, and $e$, respectively.

One can also obtain the analog of Corollary 2, but this requires developing the notion of equivalent primes of $A_{1}$. To do this, one follows the procedure in [5] for group actions, replacing Lorenz and Passman's results on primes in $R$ and $R * G$ [3] with Cohen and Montgomery's results on primes in $A$ and $A \# k[G]^{*}$ [2]. (We note, however, that the results of [2] that we use depend on the results in [3].)

Definition. $p, q \in \operatorname{Spec} A_{1}$ are equivalent (write $p \sim q$ ) if $\Psi^{-1}(p) \cap A=$ $\Psi^{-1}(q) \cap A$.

This definition clearly gives an equivalence relation on $\operatorname{Spec} A_{1}$, but a formulation internal to $A$ will be more useful. To describe this, we need the fact $G$ acts as automorphisms on $S=A \# k[G]^{*}$, namely by $\left(a p_{h}\right)^{g}=a p_{h g}[2$, Lemma 3.3]. Then, using arguments like those in [5, Proposition 4.1], we may apply Theorems 6.2 and 7.3 of [2] to give the following characterization of equivalence.

TheOREM 6. Let $A$ be a k-algebra graded by the finite group $G$, and let $\mathscr{A}=$ $\left\{P \cap A_{1} \mid P \in \operatorname{Spec} A\right\}$. Then

(1) for each $q \in \operatorname{Spec} A_{1}$, there exists a unique $I \in \mathscr{A}$ so that $q$ is minimal over $I$;

(2) for each $I \in \mathscr{A}$, there exists only a finite number $m \leqslant|G|$ of primes of $A_{1}$ which are minimal over $I$;

(3) $q_{1} \sim q_{2}$ in $\operatorname{Spec} A_{1}$ if and only if they are both minimal over the same $I \in \mathscr{A}$;

(4) $q_{1} \sim q_{2}$ in $\operatorname{Spec} A_{1}$ if and only if $\Psi^{-1}\left(q_{1}\right)$ and $\Psi^{-1}\left(q_{2}\right)$ are in the same G-orbit in $A \# k[G]^{*}$.

We need one more lemma. It improves [1, Lemma 2.2], with an easier proof.

Lemma 2. Let $A$ be a prime PI algebra over a field $k$, and let $e$ be a nonzero idempotent in $A$. Then $\mathrm{GK}(A)=\mathrm{GK}(e A e)$.

Proof. Let $Z$ denote the center of $A$. As noted in the proof of Theorem 1, $\mathrm{GK}(A)=\mathrm{GK}(Z)$. Now the map $\Phi: A \rightarrow e A e$ given by $a \rightarrow$ eae is injective on $Z$, since $Z$ consists of regular elements, so $e Z e=e Z \cong Z$. Thus $\operatorname{GK}(Z)=\operatorname{GK}(e Z)$. 
Since $e Z \subseteq Z(e A e)$, it follows that $\mathrm{GK}(A) \leqslant \mathrm{GK}(e A e)$. But $e A e$ is a subalgebra of $A$, so $\mathrm{GK}(e A e) \leqslant \mathrm{GK}(A)$, proving the lemma.

We can now prove the analogs of [9, Theorem 2] and of Corollary 2.

Corollary 4. Let $A$ be a PI algebra over $k$, graded by the finite group $G$, and say $p \sim q$ in $\operatorname{Spec} A_{1}$. Then

(1) $\mathrm{GK}\left(A_{1} / p\right)=\mathrm{GK}\left(A_{1} / q\right)$;

(2) if also $A$ is affine, $\operatorname{cl}\left(A_{1} / p\right)=\operatorname{cl}\left(A_{1} / q\right)$.

Proof. (1) By Theorem 5, there exists $g \in G$ so that if $\mathscr{P}=\Psi^{-1}(p)$ and $\mathscr{Q}=\Psi^{-1}(q)$, then $\mathscr{Q}=\mathscr{P} g$. Thus $A \# k[G]^{*} / \mathscr{P} \cong A \# k[G]^{*} / \mathscr{Q}$, and so $\mathrm{GK}\left(A \# k[G]^{*} / \mathscr{P}\right)=\mathrm{GK}\left(A \# k[G]^{*} / \mathscr{Q}\right)$. Applying Lemma 2 to both rings with $e=\bar{p}_{1}$, we see that $\mathrm{GK}\left(A_{1} / p\right)=\mathrm{GK}\left(A_{1} / q\right)$.

As for group actions, depth is not an equivalence invariant if $A$ is not affine. For, simply use Example 1 viewed as a $\mathbf{Z}_{2}$-graded ring, as in Example 3.

We close with some general comments on the relationship between $\operatorname{Spec} A$ and $\operatorname{Spec} A_{1}$. Define the map

$$
\begin{aligned}
f: \operatorname{Spec} A & \rightarrow \operatorname{Spec} A_{1}, \\
P & \rightarrow\left\{p \mid p \text { is minimal over } P \cap A_{1}\right\} .
\end{aligned}
$$

By Theorem 5, $f$ assigns each $P \in \operatorname{Spec} A$ to an equivalence class of primes in $\operatorname{Spec} A_{1}$.

Now, $f(P)=f(Q)$ if and only if $P \cap A_{1}=Q \cap A_{1}$. What does this say about $P$ and $Q$ ? For any ideal $I$ of $A$, let $I_{G}=\Sigma_{g} \oplus I \cap A_{g} ; I$ is graded if $I=I_{G}$. A graded ideal $I$ is graded prime if $J K \subseteq I$, for $J, K$ graded ideals of $A$, implies $J \subseteq Q$ or $K \subseteq I$. It is easy to see that if $I$ is a graded ideal, then $I$ is a graded prime if and only if $I=P_{G}$, the associated graded ideal of some prime $P$ of $A$ [2, Lemma 5.1]. Now, returning to $P, Q \in \operatorname{Spec} A$, it follows from [2, Lemma 2.8] that $P \cap A_{1}=$ $Q \cap A_{1}$ if and only if $P_{G}=Q_{G}$; that is, they have the same associated graded prime.

Thus if $\operatorname{Gr} \operatorname{Spec} A$ denotes the set of graded prime ideals of $A$, then $f$ induces a bijection

$$
\tilde{f}: \operatorname{Gr} \operatorname{Spec} A \rightarrow \operatorname{Spec} A_{1} / \sim \text {. }
$$

Moreover, each graded prime $P_{G}$ determines a finite set of primes $\left\{P_{\alpha}\right\}$ with $\left(P_{\alpha}\right)_{G}=P_{G}$; the $\left\{P_{\alpha}\right\}$ are precisely the set of primes in $A$ minimal over $P_{G}$.

\section{REFERENCES}

1. J. Alev, Sur l'extension $R^{G} \hookrightarrow R$ (Séminaire d'Algèbre Paul Dubreil é Marie-Paule Malliavin, Paris, 1982), Lecture Notes in Math., vol. 1029, Springer-Verlag, New York, 1983, pp. 267-282.

2. M. Cohen and S. Montgomery, Group-graded rings, smash products, and group actions, Trans. Amer. Math. Soc. 282 (1984), 237-258.

3. M. Lorenz and D. S. Passman, Prime ideals in crossed products of finite groups, Israel J. Math. 33 (1979), 89-132.

4. M.-P. Malliavin, Dimension de Gelfand-Kirillov des algèbres a identites polynomiales, C. R. Acad. Sci. Paris Ser. A 282 (1976), 679-681.

5. S. Montgomery, Prime ideals in fixed rings, Comm. Algebra 9 (1981), 423-449.

6. Trace functions and affine fixed rings in non-commutative rings (Séminaire d'Algèbre Paul Dubriel et Marie-Paule Malliavin, Paris, 1981), Lecture Notes in Math., vol. 924, Springer-Verlag, New York, 1982, pp. 356-374. 
7. Group actions on rings: Some classical problems, Methods in Ring Theorey (F. Van Oystaeyen, ed.), Proc. 1983 NATO A.S.I., Reidel, Dordrecht, 1984, pp. 327-346.

8. S. Montgomery and L. W. Small, Fixed rings of Noetherian rings, Bull. London Math. Soc. 13 (1981), 33-38.

9. Integrality and prime ideals in fixed rings of PI rings, J. Pure Appl. Algebra 31 (1984), $185-190$.

10. D. S. Passman, Fixed rings and integrality, J. Algebra 68 (1981), 510-519.

11. L. W. Small, Rings satisfying a polynomial identity, Lecture Notes, Universitat Essen, 1980.

Department of Mathematics, University of Southern California, los Angeles, California 90089

Department of Mathematics, University of California, San Diego, California 92093 